\title{
Centrifugal Separation of Antiprotons and Electrons
}

\author{
G. Gabrielse, ${ }^{1, *}$ W. S. Kolthammer, ${ }^{1}$ R. McConnell, ${ }^{1}$ P. Richerme, ${ }^{1}$ J. Wrubel, ${ }^{1, \dagger}$ R. Kalra, ${ }^{1}$ E. Novitski, ${ }^{1}$ D. Grzonka, ${ }^{2}$ \\ W. Oelert, ${ }^{2}$ T. Sefzick, ${ }^{3}$ M. Zielinski, ${ }^{2}$ J. S. Borbely, ${ }^{3}$ D. Fitzakerley, ${ }^{3}$ M. C. George, ${ }^{3}$ E. A. Hessels, ${ }^{3}$ C. H. Storry, ${ }^{3}$ \\ M. Weel,${ }^{3}$ A. Müllers, ${ }^{4}$ J. Walz, ${ }^{4}$ and A. Speck ${ }^{5}$
}

(ATRAP Collaboration)

\author{
${ }^{1}$ Department of Physics, Harvard University, Cambridge, Massachusetts 02138, USA \\ ${ }^{2} I K P$, Forschungszentrum Jülich GmbH, 52425 Jülich, Germany \\ ${ }^{3}$ York University, Department of Physics and Astronomy, Toronto, Ontario M3J 1P3, Canada \\ ${ }^{4}$ Institut für Physik, Johannes Gutenberg Universität and Helmholtz, Institut Mainz, D-55099 Mainz, Germany \\ ${ }^{5}$ Rowland Institute at Harvard, Harvard University, Cambridge, Massachusetts 02142, USA
}

(Received 31 July 2010; published 16 November 2010)

\begin{abstract}
Centrifugal separation of antiprotons and electrons is observed, the first such demonstration with particles that cannot be laser cooled or optically imaged. The spatial separation takes place during the electron cooling of trapped antiprotons, the only method available to produce cryogenic antiprotons for precision tests of fundamental symmetries and for cold antihydrogen studies. The centrifugal separation suggests a new approach for isolating low energy antiprotons and for producing a controlled mixture of antiprotons and electrons.
\end{abstract}

DOI: 10.1103/PhysRevLett.105.213002

PACS numbers: $37.10 . \mathrm{Mn}, 52.25 . \mathrm{Xz}$

Centrifuges commonly separate both cells and nuclear isotopes within a fluid rotated at an angular rotation frequency $\omega_{r}$. The masses $m_{i}$ distribute so that larger masses are at larger radii because the centrifugal force $F_{i}=$ $m_{i} \omega_{r}^{2} \rho$ (at radius $\rho$ from the axis) is larger for larger $m_{i}$. Simultaneously trapped charged species can similarly separate within a rotating plasma [1]. When antiprotons $(\bar{p})$ were first cooled via collisions with simultaneously trapped electrons $\left(e^{-}\right)$[2], the electron density, number, and temperature conspired with a high magnetic field to prevent centrifugal separation [3]. Centrifugal separation of much colder, laser-cooled ions was observed when these were used (instead of $e^{-}$) to cool either another ion species (e.g., [4]) or positrons $\left(e^{+}\right)$[5]. The separated ion distributions were directly imaged using decay photons that followed laser excitation.

Neither lasers nor photon imaging can be used to establish and detect the centrifugal separation of trapped $e^{-}$and $\bar{p}$. The predicted separation has not been observed for this basic two-component plasma system. This is surprising given that collisional electron cooling of $\bar{p}$ [2] is routinely used as the only method to cool $\bar{p}$ to cryogenic temperatures. (Collisions with any other matter particle would annihilate the $\bar{p}$.) Electron cooling of $\bar{p}$ made possible the most stringent test of $C P T$ invariance with a baryon system [6] (a comparison of the charge-to-mass ratios of the $\bar{p}$ and $p$ ) and will be required to realize a $10^{6}$-fold improved measurement of the $\bar{p}$ magnetic moment [7]. Electron-cooling is what makes it possible to accumulate trapped $\bar{p}$ [8] for producing and studying all cold $\bar{H}$ atoms (e.g., $[9,10]$ ), and it enables the production of low energy $\bar{p}$ beams for collision studies [11].

This Letter reports the observation of centrifugal separation of trapped $\bar{p}$ and $e^{-}$. The separation is large and unavoidable for the large electron number $\left(N \sim 10^{8}\right)$, large electron density $\left(n \sim 10^{8} / \mathrm{cm}^{3}\right)$, and modest magnetic fields (down to $B \sim 1 \mathrm{~T}$ ) that are currently used along with up to $10^{7}$ cold $\bar{p}$ at ATRAP to produce and study slow $\bar{H}$. The separation is easily observed with a method that permits gently removing a well-controlled fraction of the cooling $e^{-}$from a trapped $\bar{p}$ plasma. The centrifugal separation methods should work just as well with $e^{+}$used to cool positive ions since $>10^{9} e^{+}$can now be used. (ATRAP accumulates $10^{9}$ cryogenic $e^{+}$at $8 \times 10^{5} / \mathrm{s}$ for a $20 \mathrm{mCi}$ source, which is $4 \times 10^{4} e^{+} \mathrm{s}^{-1} \mathrm{mCi}^{-1}$.)

The gold-plated, oxygen-free electrical grade copper trap electrodes used to simultaneously store $\bar{p}$ and $e^{-}$ [Fig. 1(a)] have a $B=1-3.7 \mathrm{~T}$ magnetic field directed along their axis $\hat{\mathbf{z}}$ (horizontal in the figure but vertical in reality). The five cylindrical electrodes used to demonstrate separation are part of a stack of nearly 40 electrodes (represented fully in [12]). An $80 \mathrm{eV}$ axial potential well on axis [Fig. 1(b)] is close to a harmonic potential [dot-dashed curve in Fig. 1(b)] when all electrodes are grounded except for $100 \mathrm{~V}$ applied to LTE3 [Fig. 1(a)]. The $10^{6} \bar{p}$ used for most of the trials reported here are accumulated from 9 pulses of $\bar{p}$ from CERN's unique Antiproton Decelerator using trapping and cooling methods $[13,14]$ that are now used for all $\bar{H}$ experiments. The $e^{-}$are photoelectrons that are trapped after they are liberated from a metal surface by intense ultraviolet pulses from an excimer laser [15]. 


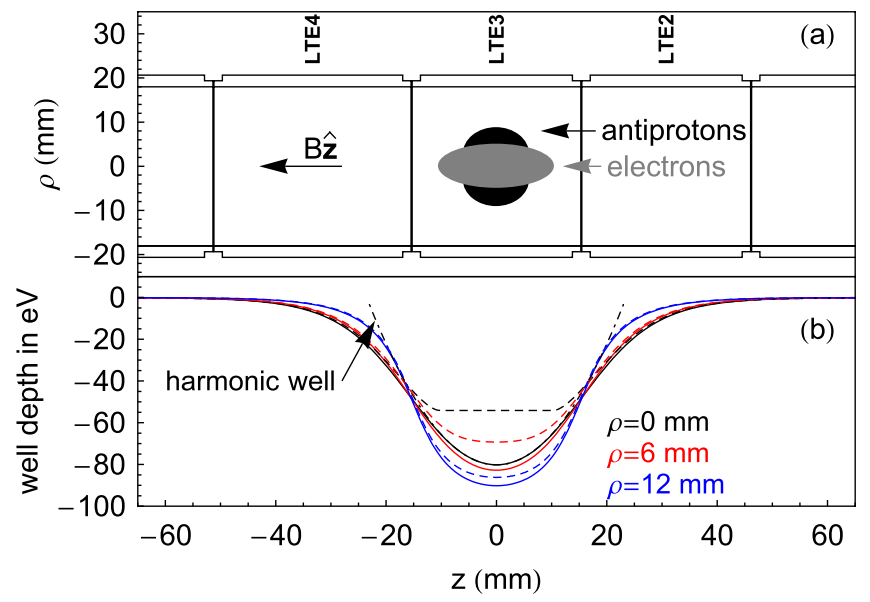

FIG. 1 (color online). (a) A $\rho=5 \mathrm{~mm}$ spheroidal $e^{-}$plasma within axially symmetric trap electrodes. The centrifugally separated $\bar{p}$ have the indicated maximum radius, but their distribution along the $\hat{\mathbf{z}}$ axis is not known. (b) $100 \mathrm{~V}$ applied to electrode LTE3 produces wells that are nearly harmonic on axis (dotdashed curve) and deepen off axis (solid curves). Potentials including the $e^{-}$space charge are shown with dashed lines.

A rotating wall potential [16] from phase-locked frequency synthesizer chips is applied to the four sections of a cylindrical ring electrode (split parallel to the trap axis every $90^{\circ}$ ). Inside, the plasma of $N=10^{8} e^{-}$experiences a radial potential well that rotates about the trap's symmetry axis. The drive strength, frequency, and duration are varied to change the rotation frequency $\omega_{r} /(2 \pi)$ of the $e^{-}$plasma between 18 and $52 \mathrm{kHz}$. Within an electrostatic quadrupole potential, a one-component plasma is spheroidal with a uniform density $n$ [17]. The rotation frequency determines the $e^{-}$density $n$ (between 4.7 and $\left.13 \times 10^{7} / \mathrm{cm}^{3}\right)$, radius $\rho(8-4 \mathrm{~mm})$, and aspect ratio $\alpha$ (1.0-2.8) for the plasma.

The plasma shape, size, and density are determined by $N$ and two measured frequencies (corresponding to the two peaks in Fig. 2). Confirmations of this method were reported earlier $[18,19]$. The axial frequency $\omega_{z}$ is for the plasma's center-of-mass oscillation along $\hat{\mathbf{z}}$, and the
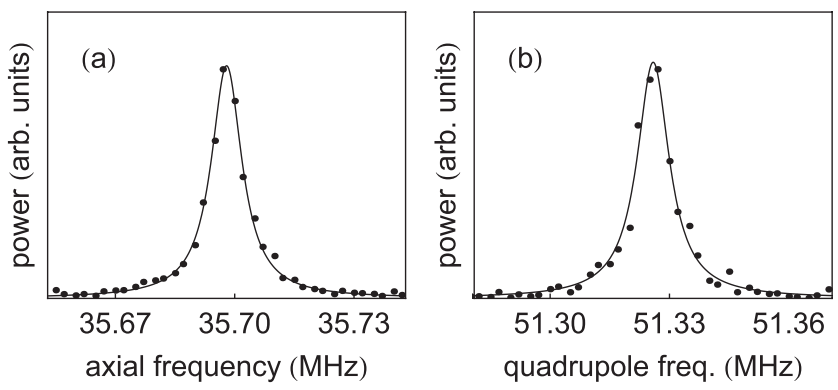

FIG. 2. Resonances that reveal the axial frequency $\omega_{z}$ of the center-of-mass (a) and the quadrupole frequency $\omega_{2}$ of the oscillation of the length (b) of an electron plasma of $10^{8}$ electrons in the $80 \mathrm{eV}$ potential well of Fig. 1. quadrupole frequency $\omega_{2}$ is for the oscillation of the length of the $e^{-}$plasma along $\hat{\mathbf{z}}$. The method (e.g., [20]) determines $\omega_{r}$ for a spheroidal plasma. (Appropriate numerical calculations can extend the method to plasmas that sample regions where the trapping potential deviates significantly from an electrostatic quadrupole [19]) The rotation (and hence the plasma shape and density) continues with the same frequency for hours.

The $\bar{p}$ might be expected to be a small perturbation on the $e^{-}$plasmas given that the $\bar{p}$ number is 100 times smaller than the $e^{-}$number. Indeed, the measured radial extent of the electron plasma agrees within measurement uncertainty with what is measured for a plasma with $10^{8}$ $e^{-}$and no $\bar{p}$, suggesting that the shape and density of the $e^{-}$plasma is the same with and without the $\bar{p}$.

Figure 3 demonstrates centrifugal separation of $\bar{p}$ and $e^{-}$. Figure 3(a) shows that $e^{-}$escape the trap before $\bar{p}$ as the applied trapping voltage is ramped down. Figure 3(b) shows that a much larger fraction of $\bar{p}$ than $e^{-}$remain in the trap. The radially centered particles escape first as the applied potential is reduced because the axial potential well is shallowest on the trap's central axis [Fig. 1(b)]. The separation [Fig. 3(a)] and measured fractions [Fig. 3(b)] do not change when the time over which the trapping depth is reduced is varied between 0.1 and $10 \mathrm{~s}$.

The released $\bar{p}$ are counted with scintillators that detect $\bar{p}$ annihilations with $75 \%$ efficiency. The released $e^{-}$can only be detected above noise levels when concentrated in small pulses whose charge can be integrated as they strike a conducting plate (biased to suppress the release of secondary $e^{-}$). The "ramps" for Fig. 3(a), and for determining the $e^{-}$remaining for Fig. 3(b), are accordingly a series of $2-\mathrm{V}$ steps separated by time delays.
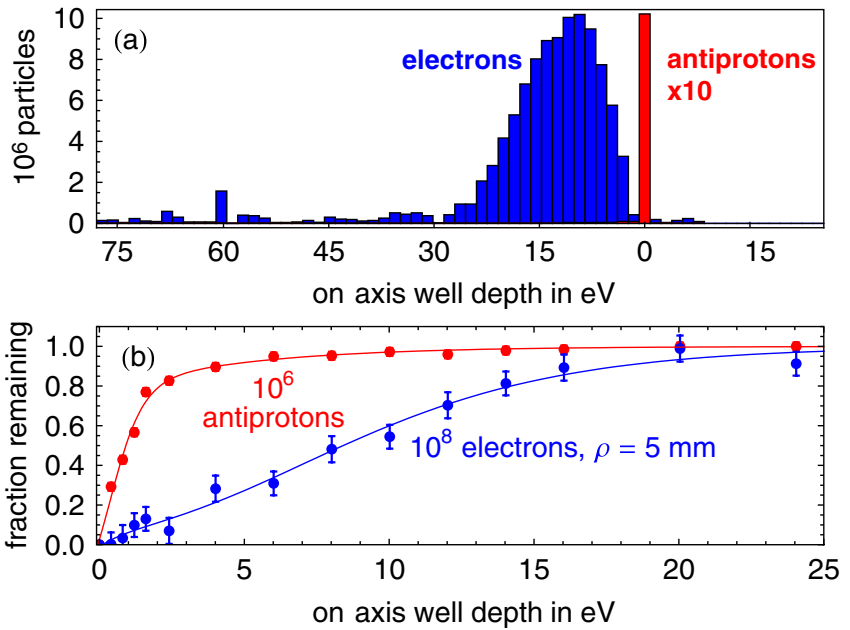

FIG. 3 (color online). (a) Number of $\bar{p}$ and $e^{-}$escaping the trap each time the applied potential is reduced by nonadiabatic, $2-\mathrm{V}$ steps. (b) Fractions of $\bar{p}$ and $e^{-}$remaining after the well depth is ramped adiabatically to the indicated value (with curves to aid the eye). The initial $e^{-}$plasma has a radius of $5 \mathrm{~mm}$ in an $80 \mathrm{eV}$ well. 
Confirmation of radial separation comes when most of the $10^{6} \bar{p}$ and none of the $10^{8} e^{-}$are lost as $B$ is reduced from 3.7 to $1 \mathrm{~T}$. The reduction expands the radial location of the particles as $B^{-1 / 2}$ (a consequence of angular momentum conservation) until the $\bar{p}$ annihilate upon striking the electrodes. In the usual symmetric gauge, the conjugate angular momentum for a charge $e$ in a $B$ field is $L_{i}=$ $(1 / 2) B e\left(\rho_{i}\right)^{2}$. It is independent of particle mass and velocity since a negligible mechanical contribution is smaller by the large ratio of the particle's cyclotron frequency and $\omega_{r}$.

It is not surprising that electrons are not lost. The sum of angular momenta for $N$ charges $e$ in a spheroid with an outer radius $\rho$ is

$$
L_{z}(\rho)=\frac{1}{5} N e B \rho^{2} .
$$

$L_{z}$ conservation thus means a fixed $B \rho^{2}$. An $e^{-}$spheroid prepared with $\rho=8 \mathrm{~mm}$ (the largest studied) grows to just over $15 \mathrm{~mm}$, well short of the $18 \mathrm{~mm}$ electrode radius.

The field at which $\bar{p}$ loss begins as $B$ is reduced (Fig. 4) suggests that the outer $\bar{p}$ radius is much larger than the radius of the electron spheroid. The deduced outer $\bar{p}$ radius decreases when the initial radius of the $e^{-}$spheroid is reduced, suggesting that the $\bar{p}$ are not uncoupled from the $e^{-}$. The superconducting solenoid's inductance limits the ramp rate to $7 \times 10^{-3} \mathrm{~T} / \mathrm{s}$, but the result shown does not change if the ramp is slowed by a factor of 2 .

A simple estimate establishes when the centrifugal separation of trapped $\bar{p}$ and $e^{-}$should take place. Equating the centrifugal energy difference for the two species, $\left(m_{p}-\right.$ $\left.m_{e}\right) \omega_{r}^{2} \rho^{2} / 2$, to $k_{B} T_{\text {sep }}$, gives the temperature below which separation should be pronounced,

$$
T_{\text {sep }} \approx \frac{m_{p} e^{2}}{8 \epsilon_{0}^{2} k_{B}}\left(\frac{n \rho}{B}\right)^{2} .
$$

The good approximation $\omega_{r} \approx e n /\left(2 \epsilon_{0} B\right) \ll \omega_{c}$ [20] is used. $T_{\text {sep }}$ ranges from 50 to $100 \mathrm{~K}$ for our $e^{-}$plasmas with

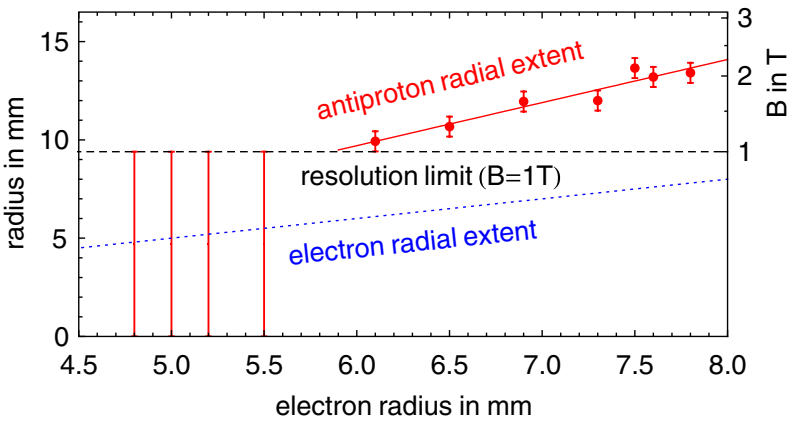

FIG. 4 (color online). Outer $\bar{p}$ plasma radius at $3.7 \mathrm{~T}$ as a function of the $e^{-}$plasma radius, deduced by ramping $B$ from 3.7 to $1 \mathrm{~T}$ and observing the value at which $\bar{p}$ annihilations begin (for $10^{6} \bar{p}$ and $10^{8} e^{-}$in a $80 \mathrm{eV}$ well). No observed annihilations for the four measurements on the left establish that the outer $\bar{p}$ extent is smaller than $9.4 \mathrm{~mm}$. The $e^{-}$plasma radius (dotted line) is included for comparison. radial extent from 8 to $4 \mathrm{~mm}$ and $B=3.7 \mathrm{~T}$. The observed separation suggests that the temperature of the $e^{-}$plasma within our $1.2 \mathrm{~K}$ trap electrodes [21] is below the range given, consistent with direct measurements of the temperature of $\bar{p}$ cooled by the electrons. Comparing to other experiments, the first $e^{-}$cooling of $\bar{p}$ [2] used many fewer $e^{-}$, a lower $e^{-}$density, and a higher $B$. The estimated $T_{\text {sep }}=1 \mathrm{~K} \mathrm{[3]}$ is lower than could be realized within $4.2 \mathrm{~K}$ trap electrodes so no centrifugal separation was expected. A recent report that there is no centrifugal separation of $\bar{p}$ and $e^{-}$is puzzling; it seems inconsistent with the reported plasma temperature and density [22].

The observation of centrifugal separation of $e^{-}$and $\bar{p}$ is the first step toward understanding this basic twocomponent plasma system. What is now needed to understand the system are predictions and measurements of the steady-state spatial distribution of $\bar{p}$.

This $\bar{p}$ distribution within the centrifugally separated plasma is crucial to the removal of $e^{-}$, and also to the distribution and temperature of the $\bar{p}$ after $e^{-}$ejection. The $\bar{p}$ distribution after $e^{-}$ejection is important because efficient $\bar{H}$ production requires matching the $\bar{p}$ distribution to the radial $e^{+}$distribution. The $\bar{p}$ temperature after $e^{-}$ ejection is important because cold $\bar{p}$ are a minimal requirement for the production of cold $\bar{H}$.

The same method to remove $e^{-}$from the $\bar{p}$ is used as was used when $e^{-}$first cooled trapped $\bar{p}$ [2] and in all cold $\bar{H}$ experiments. The depth of a trap that contains $\bar{p}$ and $e^{-}$is pulsed to $0 \mathrm{eV}$ with pulses long enough so $e^{-}$thermal velocities take them out of the well before the well is restored, but short enough that heavier $\bar{p}$ do not move much or escape. Centrifugal separation of the $\bar{p}$ and $e^{-}$ should make this pulsed ejection method more effective in that radially centered $e^{-}$can leave along the axis without colliding with $\bar{p}$ since these are at larger radii. Fewer pulses, and hence less $\bar{p}$ heating from pulsed electric fields that accelerate them, should be required to get rid of the $e^{-}$.

Conservation of $L_{z}$ for the $\bar{p}$ makes their initial radial location $\rho_{i}$ increase when $e^{-}$are pulsed out from a centrifugally separated distribution. After the electron ejection, the single component plasma of $\bar{p}$ in an electrostatic quadrupole distribution rearranges into a spheroid with radius $\rho_{p}$. This spheroid's angular momentum [from Eq. (1)] is $L_{z}\left(\rho_{p}\right)$. It equals $L_{z}$ for the $\bar{p}$ distribution just before the $e^{-}$are removed, if their ejection is faster than they can rearrange. When $\bar{p}$ are initially distributed uniformly between $\rho_{1}$ and $\rho_{2}$,

$$
\rho_{1} \leq \rho_{i} \leq \rho_{2} \rightarrow \rho_{p}=\sqrt{\frac{5}{4}\left(\rho_{1}^{2}+\rho_{2}^{2}\right)}
$$

For $\bar{p}$ between a typical $e^{-}$spheroid, $\rho_{1}=\rho_{e}=4.5 \mathrm{~mm}$, and the $\bar{p}$ outer radius measured for this spheroid, $\rho_{2}=$ $6 \mathrm{~mm}$, this results in a $\bar{p}$ spheroid with $\rho_{p}=8.4 \mathrm{~mm}$. In the limit that the $\bar{p}$ are initially at the outer boundary of an 
electron spheroid at $\rho_{e}=4.5 \mathrm{~mm}$, as might be expected, then $\rho_{p}=\sqrt{5 / 2} \rho_{e}=7.1 \mathrm{~mm}$.

This work offers a possible alternative to pulsed ejection of $e^{-}$. Figure 3(b) illustrates the alternative of removing many $e^{-}$without ejection pulses that could accelerate $\bar{p}$ and raise their temperature. This new approach may allow the $e^{-}$removal to be done slowly enough that $e^{-}$can cool away some of the $\bar{p}$ potential energy liberated as $e^{-}$are released.

The temperatures of centrifugally separated plasmas before and after one of the component species is removed is interesting and important. The long-term goal [23] of trapping $\bar{H}$ atoms for precise measurements requires very low temperature $\bar{p}$ and $e^{+}$plasmas. The number of $\bar{p}$ in the low energy tail of a Boltzmann distribution increases sharply with the distribution temperature, as $T^{-3 / 2}$. A report on work underway will detail how to use centrifugally separated plasmas to prepare $10^{6} \bar{p}$ with $T<6 \mathrm{~K}$ (without the evaporative loss that gives orders of magnitude fewer $\bar{p}$ at a comparable temperature [24]).

In conclusion, the centrifugal separation of simultaneously trapped $\bar{p}$ and $e^{-}$is clearly observed and studied. The radial separation is important given that $e^{-}$cooling of $\bar{p}$ is currently the only available method for producing cryogenic $\bar{p}$. The cooling of $\bar{p}$ is required for precision tests of fundamental symmetries and for all cold $\bar{p}$ and $\bar{H}$ studies. These studies provide some insight into and cautions for the pulse method currently used to separate $\bar{p}$ from their cooling $e^{-}$. The technique introduced to demonstrate the centrifugal separation suggests a new approach to remove the cooling $e^{-}$with less heating and with good control of the ratio of $\bar{p}$ to cooling $e^{-}$.

We are grateful to CERN for the $5-\mathrm{MeV} \bar{p}$ from its Antiproton Decelerator and to T. O'Neil for helpful comments. This work was supported by the NSF and AFOSR of the U.S., the BMBF, DFG, and DAAD of Germany, and the NSERC, CRC, CFI, and OIT of Canada. W. O. was supported in part by CERN.

*ATRAP Spokesperson. gabrielse@physics.harvard.edu

Current address: NIST, Gaithersburg, MD 20899, USA.

[1] T. M. O’Neil, Phys. Fluids 24, 1447 (1981).

[2] G. Gabrielse, X. Fei, L. A. Orozco, R. L. Tjoelker, J. Haas, H. Kalinowsky, T. A. Trainor, and W. Kells, Phys. Rev. Lett. 63, 1360 (1989).

[3] S. L. Rolston and G. Gabrielse, Hyperfine Interact. 44, 233 (1989).

[4] D. J. Larson, J. C. Bergquist, J. J. Bollinger, W. M. Itano, and D. J. Wineland, Phys. Rev. Lett. 57, 70 (1986).

[5] B. M. Jelenkovic, A. S. Newbury, J. J. Bollinger, W. M. Itano, and T. B. Mitchell, Phys. Rev. A 67, 063406 (2003).
[6] G. Gabrielse, X. Fei, L. A. Orozco, R. L. Tjoelker, J. Haas, H. Kalinowsky, T. A. Trainor, and W. Kells, Phys. Rev. Lett. 65, 1317 (1990).

[7] N. Guise, J. DiSciacca, and G. Gabrielse, Phys. Rev. Lett. 104, 143001 (2010).

[8] G. Gabrielse, N.S. Bowden, P. Oxley, A. Speck, C. H. Storry, J. N. Tan, M. Wessels, D. Grzonka, W. Oelert, G. Schepers, T. Sefzick, J. Walz, H. Pittner, and E. A. Hessels, Phys. Lett. B 548, 140 (2002).

[9] G. Gabrielse, N.S. Bowden, P. Oxley, A. Speck, C. H. Storry, J. N. Tan, M. Wessels, D. Grzonka, W. Oelert, G. Schepers, T. Sefzick, J. Walz, H. Pittner, T. W. Hänsch, and E. A. Hessels, Phys. Rev. Lett. 89, 213401 (2002).

[10] M. Amoretti et al., Nature (London) 419, 456 (2002).

[11] N. Kuroda, H. A. Torii, K. Yoshiki Franzen, Z. Wang, S. Yoneda, M. Inoue, M. Hori, B. Juhász, D. Horváth, H. Higaki, A. Mohri, J. Eades, K. Komaki, and Y. Yamazaki, Phys. Rev. Lett. 94, 023401 (2005).

[12] G. Gabrielse, P. Larochelle, D. Le Sage, B. Levitt, W. S. Kolthammer, R. McConnell, P. Richerme, J. Wrubel, A. Speck, M. C. George, D. Grzonka, W. Oelert, T. Sefzick, Z. Zhang, A. Carew, D. Comeau, E. A. Hessels, C. H. Storry, M. Weel, and J. Walz, Phys. Rev. Lett. 100, 113001 (2008).

[13] G. Gabrielse, X. Fei, K. Helmerson, S. L. Rolston, R. L. Tjoelker, T. A. Trainor, H. Kalinowsky, J. Haas, and W. Kells, Phys. Rev. Lett. 57, 2504 (1986).

[14] G. Gabrielse, Adv. At. Mol. Opt. Phys. 45, 1 (2001).

[15] B. Levitt, G. Gabrielse, P. Larochelle, D. Le Sage, W. S. Kolthammer, R. McConnell, J. Wrubel, A. Speck, D. Grzonka, W. Oelert, T. Sefzick, Z. Zhang, D. Comeau, M. C. George, E. A. Hessels, C. H. Storry, M. Weel, and J. Walz, Phys. Lett. B 656, 25 (2007).

[16] X. P. Huang, F. Anderegg, E. M. Hollmann, C. F. Driscoll, and T. M. O'Neil, Phys. Rev. Lett. 78, 875 (1997).

[17] D. H. E. Dubin, Phys. Fluids B 5, 295 (1993).

[18] P. Oxley, N. S. Bowden, R. Parrott, A. Speck, C. Storry, J. N. Tan, M. Wessels, G. Gabrielse, D. Grzonka, W. Oelert, G. Schepers, T. Sefzick, J. Walz, H. Pittner, T. W. Hänsch, and E. A. Hessels, Phys. Lett. B 595, 60 (2004).

[19] A. Speck, G. Gabrielse, P. Larochelle, D. Le Sage, B. Levitt, W. S. Kolthammer, R. McConnell, J. Wrubel, D. Grzonka, W. Oelert, T. Sefzick, Z. Zhang, D. Comeau, M. C. George, E. A. Hessels, C. H. Storry, M. Weel, and J. Walz, Phys. Lett. B 650, 119 (2007).

[20] J. J. Bollinger, D. J. Heinzen, F. L. Moore, W. M. Itano, D. J. Wineland, and D. H. E. Dubin, Phys. Rev. A 48, 525 (1993).

[21] J. Wrubel et al. (ATRAP Collaboration) (to be published).

[22] G. B. Andresen et al. (ALPHA Collaboration), Phys. Rev. Lett. 100, 203401 (2008).

[23] G. Gabrielse, in Fundamental Symmetries, edited by P. Bloch, P. Pavlopoulos, and R. Klapisch (Plenum, New York, 1987) pp. 59-75.

[24] G. B. Andresen et al. (ALPHA Collaboration), Phys. Rev. Lett. 105, 013003 (2010). 